\title{
Microstructured sapphire shaped crystals for anitiresonant and bandgap terahertz waveguiding
}

\author{
G.M. Katyba ${ }^{1,2, *}$, N.V. Chernomyrdin ${ }^{2,3}$ K.I. Zaytsev ${ }^{2,3}$, and V.N. Kurlov ${ }^{1,2}$ \\ ${ }^{1}$ Institute of Solid State Physics of the Russian Academy of Sciences, Chernogolovka 142432, Russia \\ ${ }^{2}$ Bauman Moscow State Technical University, Moscow 105005, Russia \\ ${ }^{3}$ Prokhorov General Physics Institute of the Russian Academy of Sciences, Moscow 119991, Russia \\ *E-mail: katyba_gm@issp.ac.ru
}

Terahertz $(\mathrm{THz})$ radiation offers significant scientific and technological applications in many fields [1]; among them: security systems [2,3], medical diagnosis [4-7], non-destructive sensing of materials [8], etc. Nevertheless, the use of $\mathrm{THz}$ technologies in these demanding applications is limited by the absence of commercially-available $\mathrm{THz}$ waveguides and endoscopic systems, which are capable for the $\mathrm{THz}$ wave delivery to hardly acceptable objects with small dispersion and loss.

Recently, several types of $\mathrm{THz}$ waveguide have been developed relying on various physical principles. The oldest $\mathrm{THz}$ waveguides are based on the hollowcore metal or metal-dielectric tubes [9]. Such waveguides are characterized by relatively low propagation losses, but also feature significant dispersions. In turn, the $\mathrm{THz}$ waveguides based on plasmonic structures [10], such as single or dual metal wires, metal plates and ribbons, are convenient in manufacturing and characterized with small dispersion, low propagation and bending losses; however, they are plagued by low coupling efficiency and handling problem, which prevent a practical implementation of these waveguides. Significant progress in the $\mathrm{THz}$ waveguiding is associated with numerous developments of flexible polymer microstructured waveguide with different crosssection geometry. These waveguides realize either the anti-resonant reflecting optical waveguiding (ARROW) principle [11], or the Bragg guidance by a photonic crystal (PC) cross-section [12,13]. In such waveguides, the dispersion could be managed and the loses could be minimized by optimizing the geometry of a waveguide cross-section; however, such waveguide remain very sensitive to external perturbations and bending and, thus, are not capable for operation at high temperatures and pressures, since most of the polymers possess relatively low melting temperature and radiation resistance.

An alternative approach would be to use the $\mathrm{THz}$ waveguide and fibers, based on the crystalline material, which physical and mechanical characteristics are mainly independent from temperatures/pressures environments and from measurement conditions. In order to answer the challenging problem posed by the $\mathrm{THz}$ waveguiding, in our research work, we developed several types of $\mathrm{THz}$ waveguides based on the sapphire shaped crystal. These waveguide combines unique physical properties of sapphire (it is transparent in a broad range of electromagnetic spectrum, spanning the ultraviolet, visible and infrared bands; it has high hardness, melting point, thermal conductivity, chemical resistance, tensile strength and thermal shock resistance) and advantages of the edge-defined film-fed growth (EFG) technique (it yields fabrication of the sapphire shaped crystals with a pre-determined cross-section geometry, and an impressive volumetric and surface quality of the shaped crystal) [14-16]. We designed and fabricated two microstructured hollowcore sapphire $\mathrm{THz}$ waveguides with different crosssection geometries see Fig. 1. [17,18]. We have combined numerical analysis, using Lumerical mode solution, and experimental studies, using the transmissionmode $\mathrm{THz}$ time-domain spectroscopy, in order to demonstrate that both of the developed waveguides demonstrate advanced optical performance.

The first sapphire $\mathrm{THz}$ waveguide (see Fig. 1 (a)) possesses 7 hollow channels in its cross-section - i.e. 1 core channel and 6 cladding channels [17]. The diameter of each channel is $2.5 \mathrm{~mm}$, while the outer diameter of the waveguide is $12.0 \mathrm{~mm}$. These hollow channels form a hexagonal lattice in the waveguide cross-section with the period of $3.0 \mathrm{~mm}$. Owing to rather low number of the cladding channels in the waveguide cross-section, it realizes mainly the ARROW principle of electromagnetic waveguidance. It yields guidance of $\mathrm{THz}$ radiation in a multimode regime with a small dispersion in the frequency range of 1.00 to $1.55 \mathrm{THz}$ and the lowest propagation loss of $2.0 \mathrm{~dB} / \mathrm{m}$ at $1.45 \mathrm{THz}$.

The second sapphire $\mathrm{THz}$ waveguide (see Fig 1 (b)) possesses the outer diameter of $24.0 \mathrm{~mm}$ and much more delicate PC cross-section [18]. This waveguide contains a large hollow core with the diameter of $7.15 \mathrm{~mm}$, as well as 30 channels of the PC cladding, featuring the diameters of $1.6 \mathrm{~mm}$ and forming a hexagonal PC lattice with the period of $2.8 \mathrm{~mm}$. For this shaped crystal, we observed an efficient $\mathrm{THz}$ waveguidance with a small dispersion in the frequency range of $0.65-1.2 \mathrm{THz}$ and the lowest propagation loss of $0.01-0.03 \mathrm{~dB} / \mathrm{cm}$ around $1.2 \mathrm{THz}$. We should mention that the $\mathrm{THz}$ radiation propagates throw this waveguide in an effectively two-mode regime; thus, leading to the intermodal interference phenomenon. In Ref. [18], we proposed an analytic model describing this two-mode guidance based on a coherent superposition of the amplitude of the two interfering waves featuring distinct amplitudes and phases. This twomode guidance can be useful in the intrawaveguide interferometric sensing.

Finally, In Ref. [18], we demonstrated an opportunity for using the two-mode sapphire waveguide from Fig. 1 (b) in a high-temperature $\mathrm{THz}$ intrawaveguide interferometry of sodium nitrite $\left(\mathrm{NaNO}_{2}\right)$ film melts. In our experiment, the sapphire waveguide was 
used simultaneously as a cuvette for analyte and as a $\mathrm{THz}$ waveguide. During the $\mathrm{THz}$ wave propagation throw this waveguide, the effect of intermodal beating occurs. The interference pattern behind the output end of the waveguide forms both in the frequency domain and the spatial domain. This interference pattern strongly depends on the refractive index of the analyte placed in the waveguide hollow core. In this way, we can register the phase transitions of the $\mathrm{NaNO}_{2}$ by measuring the interference pattern at different temperatures in the range of 170 to $300^{\circ} \mathrm{C}$. The sharpest change in the interference pattern corresponds to the melting temperature of $\mathrm{NaNO}_{2}$ film. The detailed description of this experiment is presented in Ref. [18].
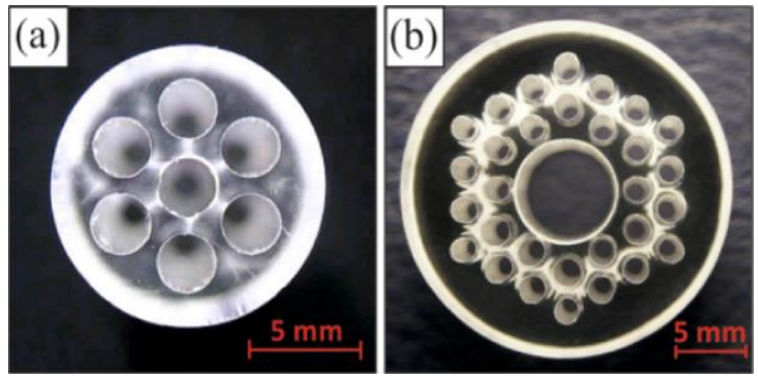

Figure 1. Cross-sections of two EFG-grown microstructured sapphire $\mathrm{THz}$ waveguides: (a) an ARROW multimode waveguide with 7 hollow channels [13]; (b) an effectively-two-mode PC waveguide with 31 hollow channels [14].

This work was supported by the Russian Foundation for Basic Research (RFBR), Projects \# 17-0800803 and 17-38-80057.

\section{References}

1. Lee, Y.-S. Principles of Terahertz Science and Technology // Springer, New York, NY, USA, 2009.

2. Dolganova, I.N., Zaytsev, K.I., Metelkina, A.A. Karasik, V.E., Yurchenko, S.O., A hybrid continuous-wave tehahertz imaging system // Review of Scientific Instruments, 2015, V. 86, No. 11, P. 113704.

3. Dolganova, I.N., Zaytsev, K.I., Yurchenko, S.O.,Karasik, V.E., Tuchin V.V., The Role of Scattering in Quasi-Ordered Structures for Terahertz Imaging: Local Order Can Increase an Image Quality // IEEE Transactions on Terahertz science and Technology, 2018, V. 8, No.4, PP. 403-409.

4. Zaytsev, K.I., Kudrin, K.G., Karasik, V.E, Reshetov, I.V., Yurchenko, S.O., In vivo terahertz spectroscopy of pigmentary skin nevi: Pilot study of non-invasive early diagnosis of dysplasia // Applied Physics Letters, 2015, V. 106, P. 053702.

5. Zaytsev, K.I., Gavdush, A.A., Chernomyrdin, N.V., Yurchenko, S.O., Highly Accurate in Vivo Terahertz Spectroscopy of Healthy Skin: Variation of Refractive Index and Absorption Coefficient Along the Human Body // IEEE Transactions on Terahertz science and Technology, 2015, V. 5, No.5, P. 817-827.

6. Chernomyrdin, N.V. Kucheryavenko, A.S. Kolontaeva, G.S., Katyba, G.M., Dolganova, I.N., Karalkin, P.A., Ponomarev, D.S., Kurlov, V.N., Reshetov, I.V., Skorobo- gatiy, M. , Tuchin, V.V., Zaytsev, K.I., Reflection-mode continuous-wave 0.15lambda-resolution terahertz solid immersion microscopy of soft biological tissues // Applied Physics Letters, 2018, V.113, No. 11, P. 111102.

7. Chernomyrdin, V.N., Gavdush; A.A., Beshplav, S.I.T.,Malakhov, K.M., Kucheryavenko, A.S., Katyba, G.M., Dolganova, I.N., Goryaynov, S.A., Karasik, V.E., Spektor, I.E., Kurlov, V.N., Yurchenko,S.O., Komandin,G.A., Potapov,A.A., Tuchin,V.V., Zaytsev,K.I., In vitro terahertz spectroscopy of gelatin-embedded human brain tumors: a pilot study // Proceedings of SPIE, 2018, V. 10716, P. 107160S

8. Yakovlev, E.V., Zaytsev, K.I., Dolganova, I.N., Yurchenko, S.O. Non-Destructive Evaluation of Polymer Composite Materials at the Manufacturing Stage Using Terahertz Pulsed Spectroscopy // IEEE Transactions on Terahertz science and Technology, 2015, V.5, No.5, P. 810-816.

9. Bowden, B., Harrington, J, A ,Mitrohanov O., Fabrication of terahertz hollow-glass metallic waveguides with inner dielectric coatings // Journal of Applied Physics, 2008. V. 93, P. 093110.

10. Wang, K., Mittleman D.M., Metal wires for terahertz wave guiding // Nature, 2004, V. 432, P. 376.

11. Chen, L.-J., Chen, H-.W., Kao, T.-F., Lu, J.-Y., Sun, $C .-K$., Low-loss subwavelength plastic fiber for terahertz waveguiding // Optics Express, 2006, V.31, No.3, P. 308-310.

11. Hassani, A., Dupuis, A. and Skorobogatiy, M., Low loss porous terahertz fibers containing multiple subwavelength holes // Applied Physics Letters, 2008, V. 92, P. 071101.

13. Ma, T., Guerboukha, H., Girard, M., Squires, A., Lewis, R., Skorobogatiy, M., 3D Printed Hollow-Core Terahertz Optical Waveguides with Hyperuniform Disordered Dielectric Reflectors // Advanced Optical Materials, 2016, V. 4, No.12, P. 2085

14. Antonov, P.I., Kurlov, V.N., A review of developments in shaped crystal growth of sapphire by the Stepanov and related techniques // Progress in Crystal Growth and Characterization of Materials, 2002, V. 44, No.2, P. 63-122.

15. Abrosimov, N.V., Kurlov, V.N., Rossolenko, S.N., Automated control of czochralski and shaped crystal growth processes using weighing techniques // Progress in Crystal Growth and Characterization of Materials, 2003, V. 46, No. 1-2, P. 1-57.

16. Katyba, G.M., Zaytsev, K.I., Dolganova, I.N., Shikunova, I.A., Chernomyrdin, N.V., Yurchenko, S.O., Komandin, G.A., Reshotov, I.V., Nesvizhevsky, V.V. and Kurlov, V.N., Sapphire shaped crystal for waveguiding, sensing and exposure applications // Progress in Crystal Growth and Characterization of Materials (accepted, 2018)

17. Zaytsev K.I., Katyba G.M., Kurlov V.N., Shikunova I.A., Karasik V.E., Yurchenko S.O., Terahertz Photonic Crystal Waveguides Based on Sapphire Shaped Crystals // IEEE Transactions on Terahertz science and Technology, 2016. V.6, No. 4, P. 576-582.

18. Katyba G.M., Zaytsev K.I., Chernomyrdin N.V. Shikunova I.A., Komandin G.A., Anzin V.B., Lebedev S.P., Spektor I.E., Karasik V.E., Yurchenko S.O., Reshetov I.V., Skorobogatiy M., Kurlov V.N. and Skorobogatiy M., Sapphire photonic crystal waveguide for terahertz sensing in aggressive environments // Advanced Optics Materials, 2018, DOI: 10.1002/adom.201800573. 